\title{
Rapid apsidal motion in eccentric eclipsing binaries: OX Cassiopeia, PV Cassiopeia, and CO Lacertae
}

\author{
P. Švaříček ${ }^{1}$, M. Wolf ${ }^{1}$, A. Claret ${ }^{2}$, L. Kotková ${ }^{3}$, L. Brát ${ }^{4}$, L. Šmelcer ${ }^{5}$, and M. Zejda ${ }^{6}$ \\ 1 Astronomical Institute, Faculty of Mathematics and Physics, Charles University Prague, V Holešovičkách 2, \\ 18200 Praha 8, Czech Republic \\ e-mail: Petr.Svaricek@seznam.cz; wolf@cesnet.cz \\ 2 Instituto de Astrofísica de Andalucía, CSIC, Apartado 3004, 18080 Granada, Spain \\ e-mail: claret@iaa.es \\ 3 Astronomical Institute, Academy of Sciences, 25165 Ondřejov, Czech Republic \\ e-mail: lenka@asu.cas.cz \\ 4 Private Observatory, Velká Úpa 193, 54221 Pec pod Sněžkou, Czech Republic \\ e-mail: brat@snezkou.cz \\ 5 Public Observatory, Vsetínská 78, 57501 Valašské Meziříčí, Czech Republic \\ e-mail: 1smelcer@astrovm.cz \\ ${ }^{6}$ Institute of Theoretical Physics and Astrophysics, Masaryk University, Kotlářská 2, 61137 Brno, Czech Republic
}

Received 6 June 2007 / Accepted 16 October 2007

\section{ABSTRACT}

\begin{abstract}
Aims. Double-lined eclipsing binaries are a traditional tool to test the capability of the stellar evolutionary models. If such binaries show apsidal motion, it is also possible to check, in addition to their absolute dimensions, some aspects of their internal structure. In order to perform this additional test, we monitored the times of a minimum of three eclipsing binaries with accurate absolute dimensions.

Methods. Approximately thirty new precise times of minimum light recorded with CCD photometers were obtained for three earlytype eccentric-orbit eclipsing binaries OX Cas $(P=2.49, e=0.041)$, PV Cas (1.75, 0.032), and CO Lac (1.54, 0.029). O-C diagrams were analyzed by the Lacy's method using all reliable timings found in the literature, and the elements of apsidal motion were improved. On the other hand, stellar models computed for the precise observed masses of the three systems were used as theoretical tools to compare with the observed shift in the periastron position.

Results. We confirm very short periods of apsidal motion of approximately 38.2, 91.0, and 43.4 years for OX Cas, PV Cas, and $\mathrm{CO}$ Lac, respectively. The relativistic effects are negligible, being up to $6 \%$ of the total apsidal motion rate in all systems. The corresponding observed apsidal motion rates are in good agreement with the theoretical predictions, except for the case of PV Cas, whose components seem to be more mass concentrated than the models predict.
\end{abstract}

Key words. stars: binaries: eclipsing - stars: individual: OX Cas - stars: individual: PV Cas - stars: individual: CO Lac stars: fundamental parameters - stars: early-type

\section{Introduction}

The apsidal motion in eccentric eclipsing binaries (EEB) has been used for decades to test our models of stellar structure and evolution. All eclipsing binaries analyzed here have properties that make them important "astrophysical laboratories" for studying the structure and evolution of stars. In particular, the three northern hemisphere objects with similar brightness are hot and massive eclipsing binaries with short orbital periods of about 2 days, those orbits have been known to be slightly eccentric $(e \leq 0.04)$ and to exhibit apsidal motion.

Moreover, since the investigations of these EEBs of previous authors were based only on a limited time base and the comparison with theoretical models was based on evolutionary tracks available at that time, we decided to rediscuss the problem of apsidal motion using a substantially larger number of minimum times and new evolutionary models with new opacities, convective overshooting and mass loss rate calculated by Claret (2004, 2007).
All three systems are also included in the Atlas of $\mathrm{O}-\mathrm{C}$ Diagrams (Kreiner et al. 2001) and the recent compilation of EEBs presented by Bulut \& Demircan (2007). Our study is part of a series of papers on apsidal motion in eclipsing binaries (e.g. Wolf et al. 2004; Wolf et al. 2006). Similar studies of apsidal motion were published in the past e.g. by Giménez et al. (1986, 1987) and Lacy $(1992,1993)$.

\section{Observations of minimum light}

New CCD photometry was obtained during 2001-2006 at different observatories with the aim of securing several new, wellcovered primary and secondary minima for all variables:

- Ondřejov Observatory, Czech Republic: the 0.65-m reflector equipped with the CCD camera SBIG ST-8 or Apogee AP-7 and $V, R$ filters,

- Nicholas Copernicus Observatory and Planetarium, Brno, Czech Republic: 0.4-m or 0.2-m Newton telescopes with the CCD camera SBIG ST-7 and $R$ filter, 
Table 1. New times of minimum light.

\begin{tabular}{|c|c|c|c|c|}
\hline$\overline{~ S y s t e m ~}$ & $\begin{array}{l}\text { JD Hel.- } \\
2400000\end{array}$ & $\begin{array}{l}\text { Error } \\
\text { [day] }\end{array}$ & Epoch & Observatory \\
\hline \multirow[t]{6}{*}{ OX Cas } & 52277.5002 & 0.0005 & -693.0 & Ondřejov \\
\hline & 52572.5571 & 0.0003 & -574.5 & Ondřejov \\
\hline & 53569.47613 & 0.00008 & -174.0 & Pec \\
\hline & 53655.4199 & 0.0001 & -139.5 & Pec \\
\hline & 53920.4761 & 0.0002 & -33.0 & Ondřejov \\
\hline & 54002.6276 & 0.0001 & 0.0 & Pec \\
\hline \multirow[t]{7}{*}{ PV Cas } & 52188.33627 & 0.0003 & -825.0 & Ondřejov \\
\hline & 52280.2588 & 0.0002 & -772.5 & Ondřejov \\
\hline & 52530.57656 & 0.0001 & -629.5 & Ondřejov \\
\hline & 53632.4703 & 0.0001 & 0.0 & Pec \\
\hline & 53709.4903 & 0.0003 & 44.0 & Valašské \\
\hline & 53913.4507 & 0.0005 & 160.5 & Ondřejov \\
\hline & 53947.5540 & 0.0002 & 180.0 & Ondřejov \\
\hline \multirow[t]{14}{*}{$\mathrm{CO} \mathrm{Lac}$} & 52185.5017 & 0.0002 & -950.5 & Ondřejov \\
\hline & 52505.4811 & 0.0002 & -743.0 & Ondřejov \\
\hline & 52549.4613 & 0.0004 & -714.5 & Ondřejov \\
\hline & 52856.36009 & 0.00007 & -515.5 & Ondřejov \\
\hline & 52900.2873 & 0.0002 & -487.0 & Ondřejov \\
\hline & 53516.4236 & 0.0003 & -87.5 & Ondřejov \\
\hline & 53543.3891 & 0.0001 & -70.0 & Ondřejov \\
\hline & 53607.41302 & 0.00008 & -28.5 & Ondřejov \\
\hline & 53613.5822 & 0.0001 & -24.5 & Brno \\
\hline & 53651.34425 & 0.00008 & 0.0 & Brno \\
\hline & 53746.2111 & 0.0001 & 61.5 & Ondřejov \\
\hline & 53913.5204 & 0.0002 & 170.0 & Ondřejov \\
\hline & 53920.4801 & 0.0003 & 174.5 & Ondřejov \\
\hline & 53987.5467 & 0.0005 & 218.0 & Brno \\
\hline
\end{tabular}

- Valašské Meziříčí Observatory, Czech Republic: 0.3-m Celestron Ultima telescope with the CCD camera SBIG ST-7, and $I$ filter, and

- Private observatory of L.B. at Pec pod Sněžkou, Czech Republic: 0.2-m Cassegrain telescope with the CCD camera SBIG ST-8 and $R$ filter.

The CCD measurements in the Brno, Ondřejov, Pec and Valašské Meziříčí observatories were flat-fielded via sky exposures taken at dusk or dawn. Several comparison stars were chosen on the same frame as the variables. No correction for differential extinction was applied, due to the proximity of the comparison stars to the variable and the resulting negligible differences in air mass. See also http://nyx.asu.cas.cz/

lenka/dbvar/ for observational circumstances.

The new times of primary and secondary minima and their errors were generally determined by fitting the light curves by means of polynomials. All times given in Table 1 were also corrected from UTC to the uniform and stable time scale TAI by means of data from BIPM. Usually, only the bottom parts of the eclipses were used. All epochs in Table 1 are calculated from the light elements given in the text, the other columns are selfexplanatory.

\section{Apsidal motion analysis}

The apsidal motion in all systems was studied using the method described in Lacy (1992). This method is based on the assumption that the orbital path of the secondary star with respect to the primary one can be approximated as being an ellipse whose major axis (line of apsides) is advancing in the plane of the ellipse at a constant rate. One of the key relationships of the Lacy method is

$\delta=\frac{\left(1-e^{2}\right) \sqrt{1-\sin ^{2} i \cos ^{2} \theta}}{1-e \sin (\theta-\omega)}$,

which expresses projected angular separation between centers of the stars. Maximum eclipse will occur when $\delta$ is a minimum with respect to the angle $\theta$, where

$\theta=v+\omega-\frac{\pi}{2}$,

and $v$ and $\omega$ denote the true anomaly of the secondary star and argument of periastron resp. The geometrical meaning can be found in Giménez \& García-Pelayo (1983). By minimizing Eq. (1) we obtain the true anomaly $v$, which is then used to calculate the eccentric anomaly $\mathcal{E}$. It is related to the last periastron passage through the Kepler's equation in the following form

$\left(t-t_{0}\right)=(\mathcal{E}-e \sin \mathcal{E}) \frac{P_{\mathrm{S}}}{2 \pi}$.

The predicted time of eclipse is then

$T=T_{0}+m P_{\mathrm{A}}+\left(t-t_{0}\right)$,

where $m$ denotes the number of periastron passages since $T_{0}$, which is time of the first periastron passage prior to the primary minimum at $t_{0}$. Integer $m$ is calculated by means of rough estimation of the eclipse time

$T=P_{\mathrm{S}} E+t_{0}$.

Further iterations are necessary, until the difference between two consecutive iterations becomes sufficiently small. We refer readers to Lacy (1992) for more detailed information. Once the set of theoretical times of eclipses is known, we proceed in the same general way as in case of any other non-linear least squares problem. We also use the Levenberg-Marquardt method. Initial estimates of the parameters are necessary. For this purpose we used the values derived in previous papers.

There are five independent variables $\left(t_{0}, P_{\mathrm{A}}, e, \dot{\omega}, \omega_{0}\right)$ determined in our computation. The periastron position $\omega$ is defined by the linear equation

$\omega=\omega_{0}+\dot{\omega} E$,

where $\dot{\omega}$ is the rate of periastron advance, and the position of periastron for the zero epoch $t_{0}$ is denoted as $\omega_{0}$. The relation between the sidereal and the anomalistic period, $P_{\mathrm{S}}$ and $P_{\mathrm{A}}$, is given by

$P_{\mathrm{S}}=P_{\mathrm{A}}\left(1-\dot{\omega} / 360^{\circ}\right)$

and the period of apsidal motion by

$U=360^{\circ} P_{\mathrm{A}} / \dot{\omega}$.

We collected all reliable times of minimum light available in the literature. All times of minima were used with the weights in our computation. Our weight is equal to the reciprocal value of the mean squared error of eclipse time normalized by the sum of all weights.

\subsection{OX Cas}

The detached and double-lined eclipsing binary OX Cassiopeiae (also BD+600169, HIP 5391, FL 79; $V_{\max }=9^{m} .92 ;$ $\mathrm{Sp} . \mathrm{B} 2 \mathrm{~V}+\mathrm{B} 2 \mathrm{~V})$ is a relatively well-known eclipsing binary with an eccentric orbit $(e=0.04)$ and a short orbital period 
Table 2. Apsidal motion elements for OX Cas, PV Cas and CO Lac.

\begin{tabular}{ccccc}
\hline \hline Parameter & Unit & OX Cas & PV Cas & CO Lac \\
\hline$t_{0}$ & HJD & $2454002.62522(1)$ & $2453632.46962(2)$ & $2453651.34483(4)$ \\
$P_{\mathrm{S}}$ & days & $2.4893467(4)$ & $1.7504698(9)$ & $1.54220727(18)$ \\
$P_{\mathrm{A}}$ & days & $2.4897913(3)$ & $1.7505620(7)$ & $1.54235744(13)$ \\
$e$ & - & $0.04147(5)$ & $0.03248(14)$ & $0.02888(2)$ \\
$\dot{\omega}$ & deg cycle & $0.06430(4)$ & $0.01896(14)$ & $0.03505(3)$ \\
$\dot{\omega}$ & deg yr ${ }^{-1}$ & $9.433(6)$ & $3.956(29)$ & $8.300(7)$ \\
$\omega_{0}$ & deg & $31.9(1)$ & $324.9(4)$ & $42.5(1)$ \\
$U$ & years & $38.16(2)$ & $91.0(7)$ & $43.37(4)$ \\
$n_{\mathrm{c}}$ & $\%$ & 92.0 & 52.0 & 94.5 \\
\hline
\end{tabular}

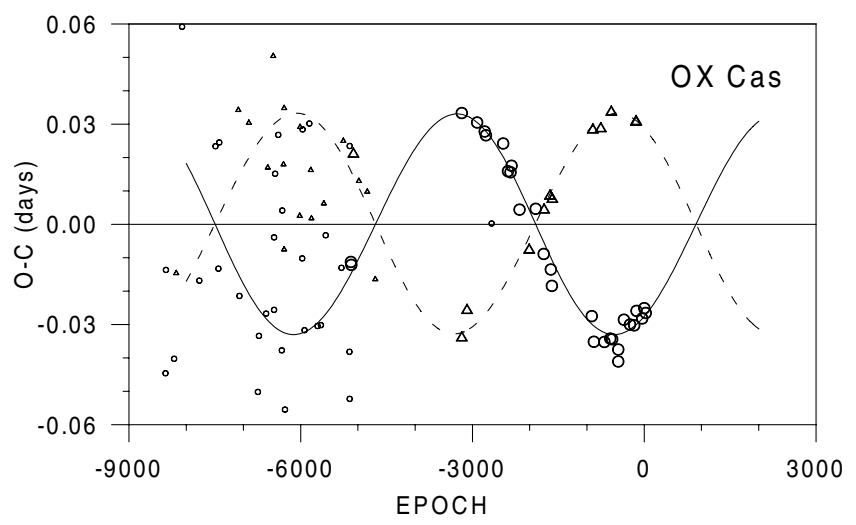

Fig. 1. The $\mathrm{O}-\mathrm{C}$ residuals for the times of minimum of $\mathrm{OX}$ Cas. The continuous and dashed curves represent predictions for the primary and secondary eclipses, respectively. The individual primary and secondary minima are denoted by circles and triangles, respectively. Larger symbols correspond to the photoelectric or CCD measurements, which were used in the calculations.

of about 2.5 days. It was first discovered to be a variable star by Reim (1957). The next photographic light curves were obtained by Geyer (1958) and later by Filin (1962). Frazier \& Hall (1975) obtained a first photoelectric light curve and detected the secondary minimum shifted relative to phase 0.5 . Schiller \& Milone (1987) obtained first spectroscopic orbit for OX Cas, from which they determined a mass ratio of 0.88 . They also derived precise absolute dimensions of the components:

$M_{1}=7.2 \pm 0.5 M_{\odot}, M_{2}=6.3 \pm 0.5 M_{\odot}$,

$R_{1}=4.69 \pm 0.3 R_{\odot}, R_{2}=4.22 \pm 0.3 R_{\odot}$.

The apsidal motion was first detected by Khaliullin et al. (1987), who obtained the rate of rapid apsidal motion of $9.1 \mathrm{deg} /$ year. A detailed study of the apsidal motion of OX Cas was later carried out by Wolf et al. (1997); see also a history of work on this binary in this paper. The possible membership of OX Cas in the open cluster NGC 381 was excluded by Crinklaw \& Talbert (1988). The epochs were calculated using the following new linear light elements

Pri. Min. = HJD $2454002.62755+2.489329 \cdot E$.

In addition to those timings given in Table 1 and in Wolf et al. (1997, their Table 2), we have added the new times of minimum obtained recently by many observers and collected in Bulut \& Demircan (2003), Agerer \& Hübscher (2003), Nelson (2003), Hübscher (2005), Hübscher et al. (2005) and Bíró et al. (2006).

A total of 41 reliable times of minimum are now available, of which 30 correspond to primary eclipses and 11 to secondary eclipses. The computed apsidal motion elements and the internal errors of the least-squares fit (in brackets) are given in Table 2. In this table, $P_{\mathrm{S}}$ denotes the sidereal period, $P_{\mathrm{A}}$ the anomalistic period, $e$ represents the eccentricity, and $\dot{\omega}$ is the rate of periastron advance (in degrees per cycle or in degrees per year). The zero epoch is given by $t_{0}$, and the corresponding position of the periastron is represented by $\omega_{0}$. The index $n_{\mathrm{c}}=\Delta T / U$ expressing the coverage of the apsidal motion period by precise photoelectric timings is also given.

The $\mathrm{O}-\mathrm{C}$ residuals for all times of minimum with respect to the linear part of the apsidal motion equation are shown in Fig. 1. The non-linear predictions, corresponding to the fitted parameters, are plotted as continuous and dashed curves for primary and secondary eclipses, respectively. Note that the differences of $\mathrm{O}-\mathrm{C}$ values from the apsidal motion are substantially larger than the standard errors of many observed times of minimum.

\subsection{PV Cas}

The double-lined and detached eclipsing binary PV Cassiopeiae (also HD 240208, BD+58 2554, FL 3470; $V_{\max }=9^{m} .86$; Sp. B9.5V + B9.5V) is a relatively bright binary system with a slightly eccentric orbit $(e=0.03)$. It was discovered to be variable star photographically by Geyer (1955). Ibanoglu (1974) made a photometric study of PV Cas and derived precise period, an orbital eccentricity $e=0.031 \pm 0.009$, and geometric parameters of the system. Other analyses of extensive series of photometric observations have been carried out by Popper \& Etzel (1981) and by Sezer et al. (1983) with similar results. Giménez \& Margrave (1982) first determined the period of apsidal motion to be $U=91 \pm 7$ and the eccentricity $e=$ $0.0322 \pm 0.0005$. Krzesinski et al. (1993) derived an apsidal motion rate of $(0.0178 \pm 0.0009) \mathrm{deg} /$ cycle, slightly longer than that obtained by Giménez \& Margrave (1982). Precise absolute dimensions and masses of the components of PV Cas were taken from Popper (1987)

$M_{1}=2.82 \pm 0.05 M_{\odot}, M_{2}=2.76 \pm 0.06 M_{\odot}$, $R_{1}=2.297 \pm 0.021 R_{\odot}, R_{2}=2.256 \pm 0.016 R_{\odot}$.

Recently, Yildiz (2005) showed that in this system only stellar models with differential rotation are in agreement with observation of apsidal motion and the synchronous rotation of components. He also derived the apsidal period of $U=91.3 \pm 1.7$ years. Some of his results will be discussed in the Conclusions.

Since the above-mentioned papers were published, a substantial number of new times of minima has been obtained, which allowed us to reduce the observational uncertainties. Besides those times given in Table 1 and listed in Wolf (1995, his Table 2) and Barembaum \& Etzel (1995, their Table 4), we have added the numerous new times published in Jordi et al. (1996), Lacy et al. (1998), Bíró et al. (1998, 2006, 2007), 


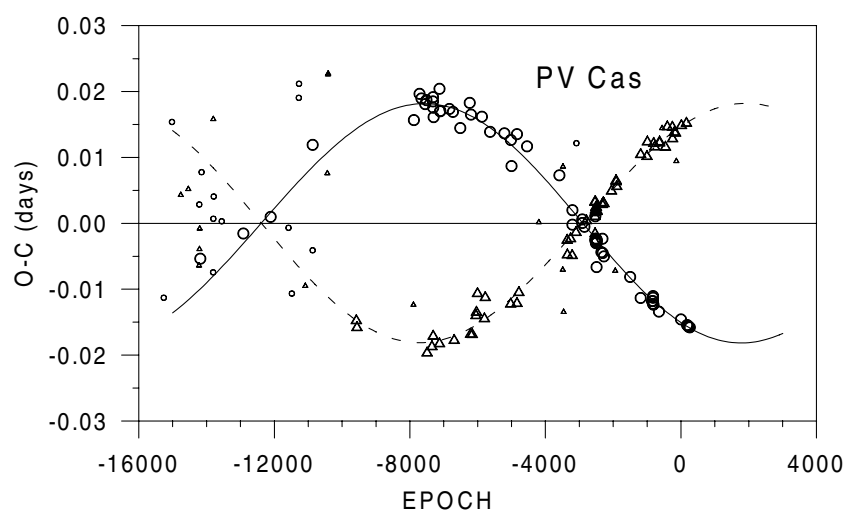

Fig. 2. O-C diagram for PV Cas. See caption for Fig. 1.

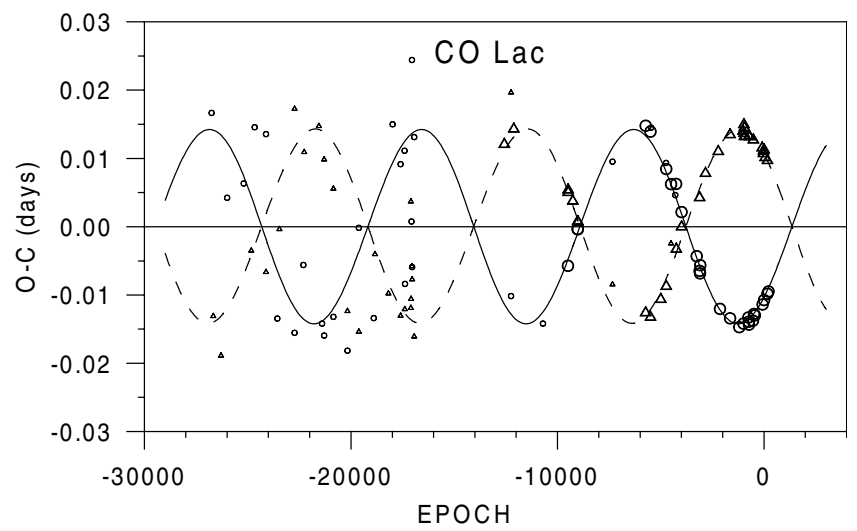

Fig. 3. O-C graph for CO Lac. See caption to Fig. 1.

Agerer \& Hübscher (2001, 2003), Nelson (2002), Hübscher (2005) and Diethelm (2005). The epochs in Table 1 were computed according to the following new ephemeris

Pri.Min. = HJD $2453632.47031+1.75046986 \cdot E$.

A total of 119 photoelectric times of minimum light were used in our analysis, including 58 secondary eclipses. The orbital inclination was adopted to be $i=85^{\circ} .37$, based on the photometric analysis of Popper (1987). The computed apsidal motion parameters and their internal errors of the least-squares fit are given in Table 2. The O-C diagram is shown in Fig. 2. The original photographic times of minima obtained by Geyer (1955) and Perova (1957), which were not taken into consideration are also plotted.

\subsection{CO Lac}

The detached eclipsing binary CO Lacertae (also HD 240058 , $\mathrm{BD}+56^{\circ} 2857$, HIP 112 436, FL 3428; $V_{\max }=10^{\mathrm{m}} .5$; Sp. B9V) is another well-known binary with a relatively rapid apsidal motion and a short orbital period of about 1.54 days. It was discovered as a variable star by Zonn (1933). Uitterdijk (1934) first suggested an apsidal motion with a period of about 40 yeras. The apsidal motion was later discussed by Zonn (1950), Semeniuk (1967), Mossakovskaya \& Khaliullin (1987) - who also discussed the possibility of the presence of a third body in this system - and Wolf (1994). The photometric elements were obtained by Mezzetti et al. (1980) and Wilson \& Woodward (1983). Recently, Švaříček (2006) in his apsidal motion study obtained relatively precise values of the eccentricity $e=0.0289 \pm 0.0002$ and the period of apsidal motion $U=43.37 \pm 0.04$ years.
Absolute dimensions and masses of the components of CO Lac were taken from Claret \& Giménez (1993)

$M_{1}=3.13 \pm 0.30 M_{\odot}, M_{2}=2.75 \pm 0.30 M_{\odot}$,

$R_{1}=2.76 \pm 0.03 R_{\odot}, R_{2}=2.49 \pm 0.06 R_{\odot}$.

The epochs were calculated using the new linear ephemeris

Pri.Min. $=$ HJD $2453651.34418+1.5422075 \cdot E$.

We include all times of minimum light collected in Wolf (1994, his Table 2), Agerer \& Hübscher (1997, 1998, 2003), Nelson (2001), Ak \& Filin (2003), Krajci (2005), Hübscher (2005), Diethelm (2006) and our new times given in Table 1. A total of 65 reliable times of minimum are now available, of which 33 correspond to primary eclipses and 32 to secondary eclipses. The orbital inclination was adopted to be $i=85^{\circ} .35$, based on the photometric analysis of Mezzeti et al. (1980). The computed apsidal motion parameters and the internal errors of the leastsquares fit are given in Table 2. The $\mathrm{O}-\mathrm{C}$ diagram is shown in Fig. 3. The original times of minimum obtained by Zonn (1933), Uitterdijk (1934) and Dugan \& Wright (1939), which were not taken into account in the calculation, are also plotted.

\section{Internal structure constant}

Observations of binary systems allow us to determine the internal structure constant (ISC), $k_{2}$, which is related to the variation of density within the star and is an important parameter of stellar evolution models. It is best studied in binary systems with eccentric orbits which show apsidal motion. The observed average value of $\bar{k}_{2 \text {,obs }}$ is given by

$\bar{k}_{2, \mathrm{obs}}=\frac{1}{c_{21}+c_{22}} \frac{P_{\mathrm{A}}}{U}=\frac{1}{c_{21}+c_{22}} \frac{\dot{\omega}_{\mathrm{N}}}{360}$,

where $c_{21}$ and $c_{22}$ are functions of the orbital eccentricity, fractional radii, the masses of the components, and the ratio between rotational velocity of the stars and Keplerian velocity (Kopal 1978). We also assume that the component stars rotate pseudosynchronously with the same angular velocity as the maximum orbital value at periastron. Taking the value of the eccentricity and the masses of the components into account, we have to subtract a relativistic correction $\dot{\omega}_{\text {rel }}$ (Giménez 1985):

$\dot{\omega}_{\text {rel }}=5.45 \times 10^{-4} \frac{1}{1-e^{2}}\left(\frac{M_{1}+M_{2}}{P_{\mathrm{A}}}\right)^{2 / 3}$,

where $M_{i}$ denotes the individual masses of the components in solar units and $P_{\mathrm{A}}$ is the anomalistic orbital period in days. The values of $\dot{\omega}_{\text {rel }}$ and the resulting mean internal structure constants $\bar{k}_{2, \text { obs }}$ for all systems are given in Table 3 . Since the relative radii of the component stars are sufficiently large, the relativistic effect makes only a small contribution up to $6 \%$ of the total apsidal motion rate. The uncertainty in the eccentricity was neglected due to its very small value and the presence of its higher terms in equations. Theoretical values $k_{2 \text {,theo }}$ were computed using new evolutionary models according to given masses and chemical composition (Table 3). New tables of opacities, convective overshooting and mass loss rate (Claret 2004, 2007) are involved in these models. Because the systems in this study are not evolved or slightly evolved binaries on the main sequence, we were able to fit the evolution of $k_{2 \text {,theo }}$ by means of a simple polynomial of 2 nd and 4 th order, respectively. The final value of $k_{2 \text {,theo }}$ is

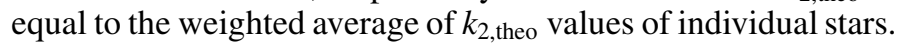


Table 3. Adopted physical properties of the binary components and the internal structure constant.

\begin{tabular}{lcccc}
\hline \hline Parameter & Unit & OX Cas & PV Cas & CO Lac \\
\hline$M_{1}$ & $M_{\odot}$ & $7.2(5)$ & $2.82(6)$ & $3.13(30)$ \\
$M_{2}$ & $M_{\odot}$ & $6.3(5)$ & $2.76(5)$ & $2.75(30)$ \\
$r_{1}$ & - & $0.2560(16)$ & $0.212(2)$ & $0.250(1)$ \\
$r_{2}$ & - & $0.2304(17)$ & $0.208(1)$ & $0.210(2)$ \\
$i$ & deg & $84.15(25)$ & $85.37(11)$ & $85.35(31)$ \\
\hline Source & & Crinklaw \& & Popper $(1987)$ & Claret \& \\
& & Etzel (1989) & & Giménez (1993) \\
\hline$\dot{\omega}_{\text {rel }}$ & deg cycle ${ }^{-1}$ & $0.00169(6)$ & $0.00118(1)$ & $0.00133(6)$ \\
$\dot{\omega}_{\text {rel }} / \dot{\omega}$ & $\%$ & 2.6 & 6.4 & 3.8 \\
$(X, Z)$ & & $0.65,0.004$ & $0.61,0.05$ & $0.70,0.02$ \\
$\log k_{2, \text { obs }}$ & & $-2.236(14)$ & $-2.460(18)$ & $-2.390(37)$ \\
$\log k_{2, \text { theo }}$ & & -2.221 & -2.327 & -2.400 \\
\hline
\end{tabular}

The weights are calculated from orbital elements and other system properties. The chemical composition of all systems was adopted according to the best agreement with the parameters $\log g, \log T_{\text {eff }}$ and $\log L$.

\section{Conclusions}

This study provides accurate information on the apsidal motion rates and the internal structure constants of three main-sequence early-type binary systems OX Cas, PV Cas and CO Lac. Compared to similar studies by previous authors, the database has been enlarged in time by about 1000 orbital cycles, or about $10-20 \%$ of the apsidal motion period. For OX Cas and CO Lac, the whole apsidal motion period is now almost completely covered by the precise photolectric or CCD measurements, and the resulting apsidal motion parameters could be considered as definitive. For all systems, a slight improvement in the apsidal period has been derived in agreement with the published results of previous authors. None of the analyzed binaries presents an important relativistic contribution of up to $6 \%$ of the total apsidal motion rate. The obtained values of the $k_{2, \text { obs }}$ are compared to their theoretical values $k_{2 \text {,theo }}$, according to available models for the ISC computed for given masses and chemical compositions, see Fig. 4.

In all binaries studied, OX Cas has one of the shortest known periods of apsidal motion. Only the systems U Oph $(U=20.1 \mathrm{yr}$, Wolf et al. 2002; Vaz et al. 2007), GL Car (25.2 yr, Giménez \& Clausen 1986) and V478 Cyg (27.1 yr, Wolf et al. 2006) have shorter periods.

The value of $k_{2 \text {,theo }}$ for PV Cas obtained from our stellar evolution models is in significant disagreement with the value derived from observed parameters. Yildiz (2005) claims that only rotating models - with a very fast core - are able to fit the observational properties of PV Cas, including the apsidal-motion. However, his method to introduce rotation and details on the rotational profile should be used with caution. For a more detailed discussion, we refer to the paper by Claret (2006).

From the current $\mathrm{O}-\mathrm{C}$ diagrams no indication concerning the presence of a third body in studied systems can be reached. Over an interval of precise timings we did not find significant variations on the $\mathrm{O}-\mathrm{C}$ diagrams, which could be caused by a light-time effect.

The absolute dimensions of components are known with low accuracy, for CO Lac with $10 \%$ only. More than 20 years has elapsed since the last spectroscopic studies of these binaries. Thus, it is also desirable to obtain new, high-dispersion, and

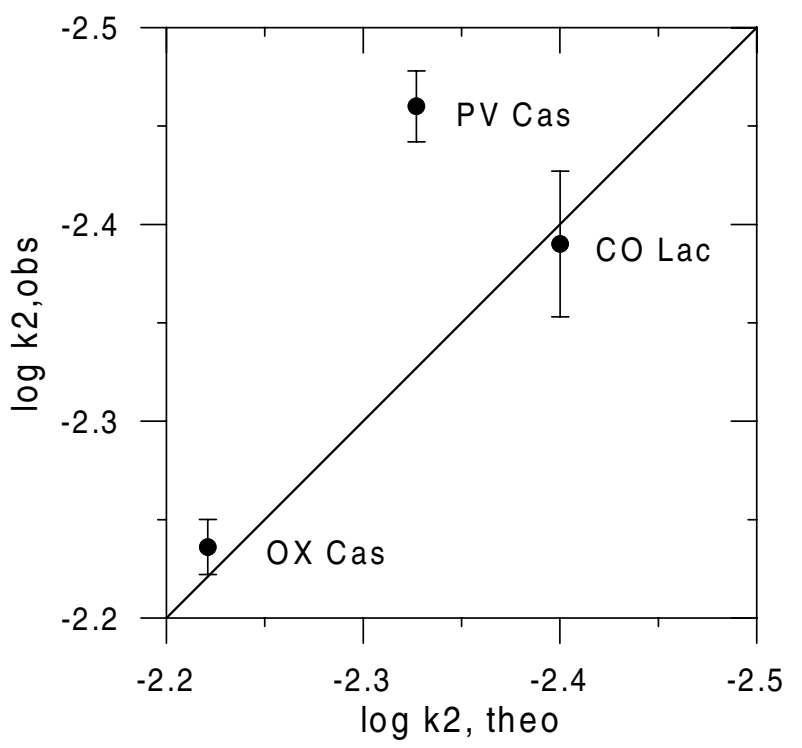

Fig. 4. A comparison between the observed and theoretical average values of $\log k_{2}$ using models of stellar evolution.

high-S/N spectroscopic observations, and apply modern disentangling methods to obtain radial velocity curves of both components for these systems.

Acknowledgements. This investigation was supported by the Grant Agency of the Czech Republic, grants Nos. 205/04/2063 and 205/06/0217. We also acknowledge the support from the Research Program MSM0021620860 of the Ministry of Education. We are also grateful to the anonymous referee for his/her suggestions which improved the manuscript. This research made use of the SIMBAD database, operated at CDS, Strasbourg, France, and of NASA's Astrophysics Data System Bibliographic Services.

\section{References}

Agerer, F., \& Hübscher, J. 1995, IBVS, 4222 Agerer, F., \& Hübscher, J. 1996, IBVS, 4383 Agerer, F., \& Hübscher, J. 1998, IBVS, 4562 Agerer, F., \& Hübscher, J. 2001, IBVS, 5016 Agerer, F., \& Hübscher, J. 2003, IBVS, 5484 Ak, H., \& Filiz, N. 2003, IBVS, 5462

Barembaum, M. J., \& Etzel, P. B. 1995, AJ, 109, 2680 Bíró, I. B., Borkovits, T., Hegedüs, T., et al. 1998, IBVS, 4555 Bíró, I. B., Borkovits, T., Csizmadia, S., et al. 2006, IBVS, 5684 Bíró, I. B., Borkovits, T., Hegedüs, T., et al. 2007, IBVS, 5753 Bulut, I., \& Demircan, O. 2003, IBVS, 5476

Bulut, I., \& Demircan, O. 2007, MNRAS, 378, 179

Claret, A. 2004, A\&A, 424, 919 
Claret, A. 2006, A\&A, 445, 1061

Claret, A. 2007, A\&A, 467, 1389

Claret, A., \& Giménez, A. 1993, A\&A, 277, 487

Crinklaw, G., \& Etzel, P. B. 1989, AJ, 98, 1418

Crinklaw, G., \& Talbert, F. D. 1988, PASP, 100, 693

Diethelm, R. 2005, IBVS, 5653

Diethelm, R. 2006, IBVS, 5713

Dugan, R. S., \& Wright, F. W. 1939, Contribution Princeton, 19, 12

Filin, A. Y. 1962, Bull. Astrophys. Inst. Tadjik, 31, 34

Frazier, T. H., \& Hall, D. S. 1975, Acta Astron., 25, 117

Geyer, E. 1955, Kleine Veröff. Remeis Sternw. Bamberg, 9

Geyer, E. 1958, Kleine Veröff. Remeis Sternw. Bamberg, 25, 8

Giménez, A. 1985, ApJ, 297, 405

Giménez, A., \& Clausen, J. V. 1986, A\&A, 161, 275

Giménez, A., \& Clausen, J. V. 1994, A\&A, 291, 795

Giménez, A., \& García-Pelayo, J. M. 1983, Ap\&SS, 92, 203

Giménez, A., \& Margrave, T. E. 1982, AJ, 87, 1233

Giménez, A., Clausen, J. V., \& Jensen, K. S. 1986, A\&A, 159, 157

Giménez, A., Kim, Ch.-H., \& Nha, I.-S. 1987, MNRAS, 224, 543

Güdür, N. 1978, Ap\&SS, 57, 17

Hübscher, J. 2005, IBVS, 5643

Hübscher, J., Paschke, A., \& Walter, F. 2005, IBVS, 5657

Ibanoglu, C. 1974, A\&A, 35, 483

Jordi, C., Ribas, I., \& Garcia, J. M. 1996, IBVS, 4300

Khaliullin, Kh. F., Kozyreva, V. S., \& Leontiev, S. E. 1987, Ap\&SS, 138, 361

Krajci, T. 2005, IBVS, 5592

Kreiner, J. M., Kim, C.-H., \& Nha, I.-S. 2001, An Atlas of O-C Diagrams of Eclipsing Binary Stars, Wydawnictwo Naukowe Akademii Pedagogicznej, Cracow, Poland

Kopal, Z. 1978, Dynamics of Close Binary Systems, Reidel, Dordrecht, Holland
Krzesiński, J., Pajdosz, G., \& Dróżdż, M. 1993, Ap\&SS, 204, 191 Lacy, C. H. S. 1992, AJ, 104, 2213

Lacy, C. H. S. 1993, AJ, 105, 630

Lacy, C. H. S., Clem, J. L., Zakirov, M., et al. 1998, IBVS, 4597

Mezzetti, M., Predolin, F., Giuricin, G., \& Mardirossian, F. 1980, A\&AS, 42, 15

Mossakovskaya, L. V., \& Khaliullin, Kh. F. 1987, Astron. Tsirk., 1495, 5

Müyesseroglu, Z., Gürol, B., \& Selam, S. O. 1996, IBVS, 4380

Nelson, R. H. 2001, IBVS, 5040

Nelson, R. H. 2002, IBVS, 5224

Nelson, R. H. 2003, IBVS, 5371

Perova, N. P. 1957, Perem. Zvezdy, 12, 124

Popper, D. M. 1987, AJ, 93, 672

Popper, D. M., \& Etzel, P. E. 1981, AJ, 86, 102

Reim, W. 1957, Kleine Veröff. Remeis Sternw. Bamberg, 17, 1

Schiller, S. J., \& Milone, E. F. 1987, BAAS, 19, 642

Semeniuk, I. 1967, Acta Astronomica, 17, 223

Sezer, C., Güdür, N., Gülmen, Ö., \& Sengonca, H. 1983, A\&AS, 53, 363

Švaříček, P. 2006, Open European Journal on Variable Stars, 23, 24

Uitterdijk, J. 1934, Bull. Astron. Inst. Neth., 7, 159

Vaz, L. P. R., Andersen, J., \& Claret, A. 2007, A\&A, 469, 285

Wilson, R. E., \& Woodward, E. J. 1983, Ap\&SS, 89, 5

Wolf, M. 1994, A\&A, 286, 875

Wolf, M. 1995, MNRAS, 277, 95

Wolf, M., Šarounová, L., \& Diethelm, R. 1997, A\&A, 317, 104

Wolf, M., Harmanec, P., Diethelm, R., et al. 2002, A\&A, 383, 533

Wolf, M., Harmanec, P., Šarounová, L., et al. 2004, A\&A, 420, 619

Wolf, M., Kučáková, M., Kolasa, M., et al. 2006, A\&A, 456, 1077

Yildiz, M. 2005, MNRAS, 363,967

Zonn, W. 1933, Wilno Bull., 14, 3

Zonn, W. 1950, Toruń Bull., 9, 18 